\title{
Le témoignage élaboratif d'Esther Mujawayo : quand il ne reste que les mots
}

\author{
Valérie Dusaillant-Fernandes \\ Université de Waterloo
}

Que ceux qui n'auront pas la volonté d'entendre cela se dénoncent comme complices du génocide au Rwanda. Moi, Yolande Mukagasana, devant vous et devant l'humanité, je déclare que quiconque ne veut prendre connaissance $\mathrm{du}$ calvaire $\mathrm{du}$ peuple rwandais est complice des bourreaux. Je ne veux ni terrifier, ni apitoyer et surtout pas apitoyer. Je veux seulement témoigner. Ces hommes qui m'ont fait subir les pires souffrances sans doute jusqu'à la fin de ma vie, je ne les hais ni les méprise, j'ai même pitié d'eux.

M.-F.Collard et P. Czaplinski, Rwanda 94 (48 $\mathrm{min}$ )

Commencer notre analyse sur le texte d'Esther Mujawayo par la déclaration d'une autre rescapée n'est évidemment pas fortuit. Les paroles émouvantes entrecoupées de larmes de Yolande 
Mukagasana ${ }^{1}$, prononcées il y a maintenant plus de dix ans, résonnent encore et s'imposent comme leitmotiv des récits des survivantes du génocide des Tutsi ${ }^{2}$ au Rwanda : témoigner sans haine et sans vengeance. Depuis Mukagasana, d'autres femmes victimes de l'Itsembabwoko ${ }^{3}$ prennent la plume et publient en France pour raconter leur histoire : on citera, entre autres, MarieAimable Umurerwa, Annick Kayitesi, Esther Mujawayo et, plus récemment, Scholastique Mukasonga ${ }^{4}$. Des femmes qui, selon Catherine Coquio, ont été "plus promptes » à témoigner, à rompre "l'éthos rwandais" de la discrétion sur soi-même» (p. 102). Par leur travail d'écriture, ces victimes transmettent, informent et surtout honorent la mémoire de leurs proches

1 Dans la version filmée de 2005 du spectacle, Yolande Mukagasana ne peut s'empêcher de pleurer devant le public. Elle finit son long témoignage en ouverture au spectacle du Groupov. Intitulé Rwanda 94 et présenté sur scène en 2000 au théâtre de Liège en Belgique, le spectacle, composé de témoignages, d'images, de musique et de théâtre, a fait une tournée mondiale, s'arrêtant même au Rwanda en 2004 dans le cadre de la Commémoration du dixième anniversaire du génocide.

2 Dans notre texte, nous adopterons la graphie des mots « Tutsi » et « Hutu » pour respecter le kinyarwanda, langue bantu du Rwanda. Nous omettons cependant les préfixes «ba » et «mu», qui marquent respectivement le pluriel et le singulier dans cette langue.

3 Itsembabwoko signifie " génocide » en kinyarwanda.

4 Yolande Mukagasana publie La Mort ne veut pas de moi (Paris, Fixot, 1997), N'aie pas peur de savoir (Paris, Robert Laffont, coll. "J'ai lu», 1999) et Les Blessures du silence. Témoignages du génocide au Rwanda (Arles, Actes Sud et Médecins sans frontières, 2001). Marie-Aimable Umurerwa est l'auteure de Comme la langue entre les dents (2000, Paris, L'Harmattan). Annick Kayitesi fait paraître en 2004 son livre intitulé Nous existons encore (Paris, Michel Lafon). La même année, c'est au tour d'Esther Mujawayo, avec la collaboration de Souâd Belhaddad, de se raconter dans SurVivantes : Rwanda, dix ans après le génocide (Paris, Éditions de l'Aube). En 2006, Scholastique Mukasonga apparaît sur la scène littéraire avec Inyenzi ou les Cafards (Paris, Gallimard, coll. "Continents noirs »). Mukasonga enchaîne avec d'autres ouvrages (autobiographiques ou romanesques) comme La Femme aux pieds nus (Paris, Gallimard, 2008), L'Iguifou (Paris, Gallimard, 2010), Notre-Dame du Nil (Paris, Gallimard, 2012) et Ce que murmurent les collines (Paris, Gallimard, 2014). 
(mari, enfants, frères et sœurs, parents) et des milliers d'autres Tutsi massacrés d'avril à juillet 1994. Même si, comme l'affirme Paul Ricœur, «le texte est la médiation par laquelle nous nous comprenons nous-mêmes » (p.115), ce travail consistant à coucher sur le papier les images qui les hantent nécessite un énorme effort de la part des victimes : elles doivent se heurter à plusieurs obstacles d'ordres cognitif et affectif 5 . Parfois, il faut des années, voire des décennies, pour que les langues se délient ou que le stylo glisse sur la feuille parce que le sujet traumatisé refoule consciemment son trauma comme mécanisme de défense psychique, perpétuant son enfermement dans l'oubli. Dans ces circonstances, la mise en récit de l'événement traumatique est une étape paradoxale puisqu'elle exhausse de la même manière l'envie de dire et le désir d'oublier. Heureusement pour les « témoignaires » que nous sommes, ces femmes rwandaises sont parvenues non seulement à dépasser leurs propres angoisses pour exprimer leurs épreuves personnelles, mais aussi, comme le fait Mujawayo, à lutter pour restaurer la mémoire des leurs dans une société moderne où certains osent encore nier qu'un génocide ait vraiment eu lieu au Rwanda.

Notre analyse s'intéresse au récit testimonial d'Esther Mujawayo intitulé La Fleur de Stéphanie, écrit en 2006 en collaboration avec son amie journaliste Souâd Belhaddad. Rescapée du génocide, Mujawayo relate cette période difficile où, en 2005, elle a dû faire face à Pierre et à Étienne, tueurs

\footnotetext{
5 Régine Waintrater note, d'une part, que le survivant peut douter par moments que ce qu'il a vécu s'est vraiment produit puisque les événements dépassent sa réalité. D'autre part, elle est d'avis que la mise en récit de leur expérience conduit les victimes vers des passages de leur vie difficiles à relater. Il faut que la version des faits à dire ou à écrire soit « acceptable, à la fois pour eux et pour les autres » (p. 116).
} 
«supposés» de Stéphanie, sa sœur. En effet, c'est lors de plusieurs participations aux gacaca ${ }^{6}$ qu'elle découvre petit à petit des détails sur la mort de ses proches. À l'affût de la moindre information, Mujawayo révèle que ce processus de mise en lumière de la vérité est essentiel pour la reconstruction psychique du survivant et de la nation tout entière parce qu'elle restaure les relations avec autrui et favorise un échange avec les agresseurs. Toutefois, cette démarche sociojuridique, qui insiste sur l'aveu et le pardon, apparaît aussi comme un frein à ce souhait de réconciliation tant espérée par le peuple rwandais. Dans notre étude, nous nous proposons de montrer qu'à travers un témoignage dit "élaboratif», Esther Mujawayo fait entendre sa voix traumatisée de victime, mais aussi celles de professionnelles impliquées dans le système de reconstruction nationale. Livrant ses réflexions sur la justice et sur la douleur du souvenir en utilisant divers procédés énonciatifs et discursifs, Mujawayo dévoile les limites et les conséquences (sociales, émotionnelles, psychologiques) des tribunaux gacaca. Peut-on parler de réconciliation quand certains tueurs nient ou mentent? Même si le tueur parle, comment faire face à son mensonge sans tomber dans la folie? Quelle justice paraît adéquate face à l'horreur des révélations? Partagée entre distance et emportement, entre parole et silence, entre guérison et reviviscence du trauma, Esther Mujawayo lutte avec les mots pour se construire un avenir et entretenir « une mémoire contre l'oubli » (2006, p. 234).

\footnotetext{
${ }^{6}$ Les juridictions gacaca ont été mises en place en 2001 pour juger la grande majorité des génocidaires. Sous le regard des habitants des environs, ces instances juridiques populaires et traditionnelles, dirigées par neuf sages locaux, aussi appelés "Intègres », confrontaient les rescapés du génocide aux meurtriers de leurs familles. Le 18 juin 2012, le gouvernement rwandais a fermé officiellement ces instances juridiques.
} 


\section{Le témoignage "élaboratif » d'Esther Mujawayo}

Mujawayo et Belhaddad ont déjà collaboré ensemble dans un premier récit de témoignage intitulé SurVivantes. Rwanda, dix ans après le génocide, paru en 2004 et qui posait la question de la survie et de ce que signifiait être rescapée d'un génocide. Déjà en 2004, Catherine Coquio soulignait, dans son ouvrage Rwanda. Le réel et les récits, que SurVivantes se présentait comme « un document de parole sur la parole des rescapées » (p. 119), une forme d'approche qui se retrouve dans le second ouvrage écrit par les deux femmes. Par ailleurs, La Fleur de Stéphanie se range dans la troisième catégorie de formes testimoniales distinguées par Régine Waintrater dans Sortir du génocide, catégorie qui se définit par «l'attitude du témoin par rapport à l'affect et au souvenir» (p. 140)7. Dans cette catégorie, c'est à travers les sentiments du destinataire, qu'il soit « auditeur ou témoignaire » (p. 140), que l'on peut mesurer le degré d'élaboration du traumatisme vécu. Waintrater distingue deux types de récits révélant la position affective du témoin. Il y a d'abord les témoignages dits «élaboratifs », dans lesquels le destinataire se sent libre et ne doit pas se sentir submergé par l'affect. Ces récits se caractérisent par une «souplesse narrative», c'est-à-dire l'adoption de "positions différentes par rapport à la narration et un certain équilibre entre l'histoire personnelle du sujet et les événements de

\footnotetext{
7 La première forme étant le récit testimonial professionnel ou militant dans lequel le témoin se place dans « une position d'observateur participant, qui pose un regard en surplomb sur les événements décrits » et la deuxième forme se caractérisant par le récit privé et personnel «centré sur la persécution » (p. 139-140).
} 
portée plus générale » (p. 141), et une " souplesse dialogique», favorisant une alternance du «je » au "nous», des passages descriptifs et des moments autoréflexifs ou affectifs (voir p. 141). Dans le deuxième type de récit, appelé "empêché », le témoin est immobilisé "soit dans la distance et l'évitement", soit collé «au souvenir du traumatisme» (p.142) et le témoignaire ressent de l'ennui ou une certaine contrainte face au récit du sujet témoignant ${ }^{8}$. Il ne fait aucun doute que La Fleur de Stéphanie entre dans la catégorie des récits élaboratifs. En effet, Mujawayo semble avoir déjà effectué un travail psychique sur elle-même et mis la distance nécessaire entre le trauma et le moment présent. Toutefois, rappelons que distance ne veut pas dire oubli. Le souvenir du génocide est le lieu d'une continuelle souffrance, qui se trouve ravivée par la confrontation entre la survivante et les tueurs de sa sœur lors des gacaca. Mais voyons d'un point de vue textuel et structurel comment La Fleur de Stéphanie fait partie des récits testimoniaux élaboratifs.

\section{Énonciation : pluralité des référents}

Penchons-nous en premier lieu sur l'énonciation plurielle, qui témoigne de la mobilité psychique de Mujawayo. Dans la première partie, le «je » énonciateur d'Esther s'adresse à un «tu», son amie Souâd Belhaddad, à qui elle a décidé de raconter son expérience personnelle des gacaca. La journaliste devient donc le témoignaire primaire de la confession testimoniale. Quand à la fille aînée d'Esther, Anna, présentée dans le texte par le pronom «elle», elle s'impose comme le

\footnotetext{
8 Waintrater remarque que ces témoignages sont pour la plupart des récits post-traumatiques.
} 
témoignaire secondaire, que nous qualifierions d'implicite puisque Mujawayo ne s'adresse pas directement à elle. L'auteure révèle que, déjà dans SurVivantes, elle a rendu « explicitement compte des derniers instants d'Innocent [son mari] » (2006, p. 36), dix ans après les faits, permettant ainsi à sa fille de savoir la vérité sur la mort atroce de son père. Un devoir de transmission s'imposait alors, sinon «les tueurs auraient définitivement gagné » (p.37). En outre, le pronom personnel «tu » est utilisé pour un destinataire bien particulier, la sœur d'Esther, Stéphanie, qui «attend», pour reprendre le verbe employé par la narratrice (p. 227), dans son au-delà, que toute la lumière soit faite sur ses derniers instants. Le lecteur ${ }^{9}$ se place comme le témoignaire ultime implicite en devenant, comme le précise Marie Bornand, « un partenaire actif dans la transmission d'une mémoire, dans l'élaboration d'une chaîne du témoignage » (p. 129). Puisque le livre est aussi et avant tout une offrande aux morts, une sépulture de mots dédiée à toutes les victimes de génocide (Mujawayo, 2006, p. 228), le lecteur est invité à faire partie de l'enterrement pour leur rendre un dernier hommage symbolique.

Telle la pédagogue et la thérapeute qu'elle est, Esther Mujawayo revient d'abord sur les prémices du génocide avec un certain détachement. Elle relate comme une historienne les faits qui ont marqué sa famille depuis 1959. Elle explique de la façon suivante ses premiers souvenirs : " ma mère, me portant sur le dos, a fui les massacres de Tutsi. Les voisins hutu d'une autre

\footnotetext{
${ }^{9}$ Après avoir entendu Esther Mujawayo parler à Patrick Poivre d'Arvor dans son émission «Vol de nuit » en 2006, il apparaît que le «tutoiement » chez l'auteure rwandaise n'est en aucun cas irrévérencieux, mais une forme directe, franche et explicite de communiquer, facilitant le contact avec l'interlocuteur.
} 
colline ont incendié nos maisons, volé ou tué notre bétail » (2006, p. 22). On remarquera ici l'emploi des adjectifs possessifs «ma», «nos» et "notre», grâce auxquels Muajawayo se fait la porte-parole de toute une famille persécutée. Au fil des trente premières pages, on note d'ailleurs la présence du pronom personnel «nous», qui renvoie premièrement à l'appartenance à la lignée familiale: «Des accalmies, oui, il y en a eu. Mais nous savions qu'elles n'étaient que des parenthèses qui suivaient une période de violence et en précédaient forcément d'autres.» (p. 23) L'auteure se pose comme déléguée d'un groupe familial qui n'existe plus dans sa forme originale, mais qui se doit de perdurer dans les mémoires de chacun à travers le récit de sa persécution. Par ailleurs, le pronom personnel « nous » reflète surtout l'amour parental qui lui a servi de «colonne vertébrale » lors de sa reconstruction après le génocide. Mujawayo s'explique: «un génocide, ça marche parce qu'il te marque et te possède le reste de ta vie, absolument. Un amour comme celui qui m'a allaitée te possède et te marque aussi pour le reste de ta vie. » (p. 40) Il est donc de son devoir, de sa mission même, de faire revivre les membres de sa famille par l'écriture, parce que les éradiquer du récit reviendrait à tuer l'amour qui la porte depuis plus de dix ans. C'est bien sûr un moyen inconscient de s'insérer dans une continuité générationnelle dont le sujet a besoin pour se construire malgré sa solitude, un sentiment qui découle de la « destruction irrémédiable du pacte social qui garantissait [son] existence » (Waintrater, p. 193). D’ailleurs, la pérennité de la famille est assurée dans le texte par l'emploi du pronom «nous », qui unit cette fois dans le présent Esther et ses deux sœurs, Joséphine et Marie-Josée, seules rescapées faisant partie 
de la famille: «Mes sœurs et moi [...]. Nous avons bien volontiers accepté. » (Mujawayo, 2006, p. 44)

Enfin, toujours dans cette partie du texte relatif aux événements prégénocide, lorsque Mujawayo s'attarde sur la politique de discrimination contre les Tutsi dans les discours radiophoniques ou dans les chansons, l'auteure utilise le pronom personnel «nous» en l'associant à son appartenance ethnique ${ }^{10}$. L'auteure s'exprime en ces termes: "des chansons, dont nousmêmes, Tutsi, fredonnions l'air si entraînant, si gai, avec un refrain qui appelait pourtant explicitement à notre extermination » (p. 24). Selon Dominique Maingueneau, le " "nous" permet au scripteur de demeurer dans le registre de la première personne tout en se démarquant du caractère individualisant qu'implique le “je"» (p. 30). L'auteure ne parle pas en son nom propre (je) mais, pour emprunter les termes de Maingueneau, " par une sorte de "contrat énonciatif", se pose en délégué d'une collectivité » (p. 30), d'où l'emploi du « nous » collectif.

\section{Le rapport à l'affect.}

\section{Distanciation par la description}

Comme nous l'avons mentionné, le récit testimonial élaboratif se caractérise par sa souplesse narrative, notamment du fait que le témoin peut passer de son histoire personnelle à des événements de portée plus générale. Mujawayo se fait historienne en quelques pages, soulignant les événements importants et les violences qui ont précédé le génocide de 1994,

\footnotetext{
10 Dans SurVivantes, ce pronom en vient à signifier « très concrètement les survivants tutsi » (p.11) ou les veuves qui œuvrent au sein d'AVEGA (Association des veuves du génocide d'avril), comme le remarque Catalina Sagarra (p. 12).
} 
insistant notamment sur les massacres de 1959, de 1963 et de 1973 tout comme sur les premières menaces, les insultes et la guerre entre Tutsi et Hutu en 1990 (p.31). Elle annonce objectivement aux témoignaires que, «du 7 avril au 4 juin 1994 », elle a perdu la « quasi-totalité » de sa famille, laissant "deux cent soixante-quatorze membres» dans des fosses communes au Rwanda. Ce souci de précision et de consignation des données a pour but de fournir un récit objectif et informatif qui éloigne les témoignaires de toute implication émotionnelle dans le travail psychique du témoin, car, dans ce travail de deuil en pleine élaboration, le témoin ne demande pas que l'on partage sa peine, mais que l'on soit attentif à sa vision des événements et réceptif à son partage de ses propres connaissances sur le trauma subi. Voilà pourquoi il n'existe aucune intervention émotionelle de Belhaddad. Celle-ci, dans son rôle de témoignaire explicite, facilite l'évacuation du tropplein en permettant à Mujawayo d'extérioriser « des contenus toxiques par leur portée traumatique » (Waintrater, p. 196). Un excès de manifestions émotionelles de la part de Belhaddad empêcherait Mujawayo d'accomplir son processus de deuil et la plongerait dans un état de faiblesse qui lui rappellerait sans aucun doute des situations psychiquement ingérables ressenties lors des événements de 1994.

L'écrivaine continue d'instruire ses témoignaires en leur prouvant, par des données précises, que les autorités se devaient d'accélérer le processus punitif des prisonniers et la démarche de réconciliation nationale (Mujawayo, 2006, p. 54) sous peine d'être perçues comme étant irresponsables face aux exigences des victimes. En privilégiant l'apport de statistiques et de faits historiques, Mujawayo tente de combler un manque d'informations qui fait défaut quand il s'agit du sort des 
membres de sa propre famille. Dans un souci d'exactitude quant aux événements historiques et aux sources de ses propos, l'auteure ajoute des notes en bas de page, empêchant ainsi que quelqu'un puisse contester son témoignage ou en douter. Le vocabulaire est simple; le récit, linéaire; les faits historiques, clairs et imbriqués dans l'histoire personnelle de Mujawayo. On apprend ainsi comment elle a fui son pays à feu et à sang : c'est grâce à quelques dollars qu'elle a pu être évacuée de «l'hôtel des Mille Collines, gardé par les casques bleus », puis elle s'est retrouvée à Kabuga, zone protégée par le Front patriotique rwandais (FPR), où « des anciennes collègues d'Oxfam [l']ont retrouvée et [l']ont emmenée en Ouganda» (2006, p. 39). Il semble que toutes ces informations et descriptions sont destinées particulièrement aux filles de Mujawayo puisque l'écriture, avoue-t-elle, lui permet de révéler ce qu'elle est incapable de leur dire de vive voix (p.37). Le livre devient également objet de transmission familiale, qu'il faudra passer de génération en génération pour que la mémoire des morts puisse perdurer dans le temps.

Dans le témoignage, il existe donc une dimension descriptive où le sujet s'inscrit dans une lignée de passeurs d'informations et de connaissances. Plus le témoin transmet ce qu'il sait, plus les preuves demeurent à jamais couchées sur le papier pour les générations suivantes. Porteuse d'une mémoire, Mujawayo sent qu'elle a le devoir de mettre au jour les atrocités commises par les criminels, exposant ainsi aux yeux de tous jusqu'où peut aller la barbarie. Elle a pris assez de distance pour relater à nouveau la mort de son mari, Innocent, étape qu'elle avait déjà entreprise dans le livre précédent, SurVivantes. Mais relevons que cette répétition n'est pas anodine. N'oublions pas qu'après un trauma, le sujet est victime d'une reviviscence intrusive (diurne ou 
nocturne) de l'événement, et cela, même après des années, voire des décennies. Judith Herman, dans Trauma and Recovery, atteste que " the impact of a traumatic event continues to reverberate throughout the survivor's lifecycle» (p. 211). Alors, il n'est pas anormal pour un sujet traumatisé de ressasser l'événement traumatique pour essayer de reprendre le contrôle sur le souvenir invasif. De plus, selon Marie-Laure Bardèche, la «répétition est insistance de la trace et résistance à la trace, retour et oubli du refoulé. Il y a répétition parce qu'il y a oubli. » (p. 147) En ce sens, revenir sur la mort atroce d'Innocent évite l'effacement de celui-ci dans la mémoire d'Esther et de ses filles. C'est aussi par le truchement de l'écriture une façon de le réaffirmer dans sa «dignité d'homme», car celui-ci a été déshumanisé, comme le souligne Mujawayo: «j'ai dit "déshumanisé", oui : Innocent, la veille de l'achever, on lui a coupé les tendons des pieds pour l'empêcher de s'enfuir » (2006, p. 35). Elle réhumanise également Rachel, sa sœur adoptive, en racontant ses derniers instants: « jetée vivante dans les latrines, elle s'est tant débattue au milieu des excréments qu'elle est finalement parvenue à se hisser sur le rebord en s'appuyant sur ses avant-bras. Alors, les tueurs les lui on coupés.» (p.78) On notera le ton neutre et respectueux de l'auteure: aucun adjectif qualifiant les tueurs, aucun terme trahissant son émotion. En fait, Mujawayo est en possession d'un savoir qu'elle communique, une réalité qui doit se dire pour que les derniers instants des proches ne soient plus source d'humiliation, mais qu'au contraire, débarrassés des éléments gênants, ils deviennent une revalorisation de leur être par l'écriture et le dire de leur dernière souffrance. 


\section{Emportement}

Mujawayo, tout comme les autres Tutsi, n'a jamais pensé que le système des gacaca allait fonctionner; elle exprime son doute à plusieurs reprises dans son texte : «franchement, je l'avoue, je ne voyais pas comment une telle pratique pouvait s'adapter à la situation du génocide » (p. 47). Puis elle finit par se rendre à l'évidence: "Personne, absolument personne au Rwanda n'aurait jamais imaginé que les tueurs parleraient un jour... Avec les gacaca, la parole a circulé.» (p.74) Cela dit, la libération de la parole des prisonniers s'est faite au prix d'un énorme effort sur soi demandé aux rescapés pour contenir leur l'affect.

Stimulée et démuselée, de Mujawayo fait entendre sa parole dans La Fleur de Stéphanie. Rappelons que dans le processus de guérison, il est impératif que le sujet traumatisé retrouve une voix parmi les siens d'abord, puis avec le groupe social qui l'entoure. Mujawayo donne son avis sur plusieurs aspects du processus juridique des gacaca, tant sur la confrontation avec les accusés que sur la façon dont les juges traitent les rescapés. Ceci est une étape importante, car elle permet au témoin de présenter la parole des rescapés qui, à bien des égards, a été oubliée dans cette mise en place d'une justice transitionnelle populaire.

Elle s'arrête tout d'abord sur les trois catégories instaurées par les autorités gouvernementales pour juger les prisonniers: 1) les «concepteurs du génocide» (Mujawayo, 2006, p. 52), 2) les «disciples et exécutants» et 3) «les complices et les pilleurs ». Les planificateurs du génocide ont été jugés par une cour pénale classique puisque classés comme 
des «impardonnables» (p. 52). Mujawayo s'en prend vigoureusement aux termes employés pour qualifier la deuxième catégorie de prisonniers : "les "petits" tueurs ou ceux qui sont passés à l'acte "sans intention de donner la mort" » (p. 53). Le discours de Mujawayo se fait dur et intransigeant: «je ne me résous pas à la cruauté de ces nuances de vocabulaire », «j'ai du mal, vraiment du mal avec celle-ci [la catégorie], car elle englobe la majorité des accusés », « je ne les considérerai jamais comme des "petits" tueurs » (p. 53). L’usage de la négation, doublée de l'adverbe «jamais», souligne la résolution de Mujawayo. Juste après ce passage, le ton de l'auteure devient plus sarcastique : "Va expliquer à une vieille maman coupée à la tête qu'on n'a pas eu l'intention de la tuer. » (p. 54) L'exemple donné révèle l'absurdité d'une telle catégorisation. Si, lors des gacaca, Mujawayo est polie, respecte la loi et les autorités gouvernementales, dans l'écriture, elle défie en revanche tout ce petit monde en dévoilant le ridicule de noms choisis pour les trois catégories.

Plus loin, Mujawayo s'emballe vigoureusement ${ }^{11}$ à l'écrit lorsqu'elle revient sur deux des huit règles exigées pour prendre la parole dans les gacaca, qui sont: «il ne faut pas interrompre celui qui a la parole » et « il est interdit de proférer des injures, d'être violent, de huer ou d'exhaler des menaces » (p.59). Voici comment elle réagit à ce prétendu dialogue constructif obligatoire ${ }^{12}$ qui doit s'élaborer dans les réunions gacaca :

\footnotetext{
11 Une des particularités du témoignage élaboratif est que le témoin peut faire part de ses émotions sur le récit qu'il entreprend.

12 Ce dialogue obligatoire, visant à la mise au jour de la vérité, devait s'établir entre le survivant et l'accusé, une confrontation passive, cadrée, qui
} 
L'autre a coupé les tiens, ou bien il a participé aux tueries, ou bien encore il en a été témoin sans s'y opposer, et la loi t'interdit explicitement tout affect, tout emportement. C'est-àdire que toi, tu as une envie instinctive de lui rentrer dedans, et tu ne peux même pas le faire avec les mots. (Silence). Tu vois, je te dis tout ça indignée, je m'étrangle et je peste et je m'emporte dans l'écriture comme je n'ai pas eu le droit de le faire dans la parole mais, comme tout le monde, moi aussi, je me suis conformée à la loi. (p. 59)

Soulignons dans ce passage le verbe d'action " rentrer dedans », qui accentue l'élan de colère qui anime Mujawayo. Toutefois, son désir de violence physique est freiné par une impossibilité d'aller jusqu'au bout de sa colère, d'où le silence qui prend le dessus. Même les mots écrits ne suffisent pas à définir la frustration et le stress mental qu'imposent les gacaca. Ainsi, on apprend qu'elle s' «étrangle » ou qu'elle «peste » simplement parce qu'elle en fait part. Si elle profère des paroles aigres ou injurieuses, comme l'indique le verbe "pester», celles-ci n'apparaissent pas dans ce passage. Le simple fait de dire la chose la soulage, comme si l'écriture du récit était une forme de défoulement psychique dont a besoin Mujawayo devant l'insurmontable épreuve des gacaca. Ce n'est pas tant le fait que les prisonniers ne purgeront jamais une peine de prison suffisante pour payer leurs crimes qui met en colère Mujawayo, mais cette mascarade que doit jouer le survivant devant les juges: "s'exprimer de façon factuelle, sans ajouter aucun commentaire, aucun affect» (p.64). Comment se dominer et parler du massacre des siens sans tomber dans l'émotion? Une solution, on imagine un scénario d'agression de l'accusé : «Tu fantasmes régulièrement de te retrouver face aux tueurs des

permettait à l'un de poser les questions qui le tourmentent et à l'autre de s'avouer responsable des actions violentes commises pendant le génocide. 
tiens pour les vilipender, te jeter sur eux et leur cracher ta haine, comme si cet acharnement allait permettre à la douleur de se déverser et d'enfin guérir. » (p. 79) On note ici le champ lexical de la colère physique et verbale avec les termes " vilipender », " se jeter sur », " cracher », " acharnement », une émotion et un élan vital par lesquels Mujawayo essaie de sortir d'un état épouvantable, une sorte de système d'autodéfense contre une souffrance intérieure qu'elle ne maîtrise plus. Face à la neutralité bienveillante et au manque d'empathie ou de compassion qui doivent être observés par les Intègres, Mujawayo ressent un «abandon » psychologique de la part des juges (p. 64). Alors, devant leur indifférence, elle s'abandonne elle aussi dans l'émotion scripturale.

Quand, à l'ombre d'un arbre, les accusés présumés des meurtres des membres de la famille d'Esther se présentent devant les Intègres du tribunal gacaca pour avouer les actes qu'ils ont perpétrés et faire une demande de pardon en bonne et due forme, Mujawayo tente désespérément de les convaincre de dire toute la vérité sur les massacres ${ }^{13}$. Devant Thomas, qui affirme qu'elle ment lorsqu'elle raconte des faits qui l'accusent, elle révèle qu'elle a perdu la maîtrise d'elle-même et a bondi en pointant son doigt menaçant sur lui (Mujawayo, 2006, p. 63). Cependant, elle est vite rabrouée par un membre des sages, qui ne tient pas compte de son état émotionnel de victime. Puisque la procédure en place n'est ni ouverte ni juste, cela enlève au processus de réconciliation une bonne part de sa valeur thérapeutique. Régine King, professeure adjointe en études sociales à l'Université du Manitoba et rescapée du génocide,

${ }^{13}$ N'oublions pas que s'il plaidait coupable, le prisonnier recevait une moindre peine. Quant au pardon, beaucoup de survivants l'ont perçu comme une stratégie cynique pour être libéré ou pour recevoir une remise de peine. 
affirme : "according to [her] observations from gacaca and the anecdotal stories heard from people who have participated in it, telling and listening to testimonies was not done as a form of dialogue because it did not engage the community participants who witnessed in silence» (p. 140).

Dans ce grand déballage de vérités et de mensonges, les victimes doivent faire face non seulement à leur trauma vécu douze ans plus tôt, mais aussi aux comportements légers et détachés des accusés. Braver leur rire dédaigneux, respecter leur libre circulation dans le village et entendre leurs mille et une excuses pour échapper à une lourde peine donnent à certains témoins un sentiment d'insécurité et une perte de confiance dans le système qui est censé les délivrer de leur trauma. Mujawayo a voulu affronter les tueurs de sa famille. Toutefois, bon nombre de Tutsi ont refusé de venir au gacaca de peur de représailles ou de peur de revivre des moments douloureux de leur propre vécu. Si les tribunaux gacaca ont tenté d'apporter une solution juridique au génocide, elles ont aussi essayé de mettre en place un dispositif de structures psychologiques pour les victimes parce qu'elles étaient conscientes du caractère traumatisant de ce processus ${ }^{14}$.

14 Juste après le génocide, les autorités rwandaises ont essayé tant bien que mal de mettre en place des cellules pour gérer le trauma collectif du pays juste après le génocide. En 1995, le « Ministère de la Réhabilitation a créé le Centre national du traumatisme (CNT)» (Bakansanga et al., p. 4). En 1998, l'Association rwandaise des conseillers en traumatisme (ARCT-RUHUKA) est fondée (Bakansanga et al., p. 4). De même, dès le début de la mise en place des gacaca, les associations Médecins du Monde et Ibuka-France ont conçu un dispositif de soutien psychologique pour les rescapés. Celui-ci regroupait des équipes de professionnels (des conseillers en traumatisme, un psychologue, un animateur psychosocial, un parajuriste) pouvant aider à tout moment ces rescapés (voir Mutarabayire-Schafer, 2010, p. 146). Un guide en Santé mentale dans le contexte des gacaca a alors été publié en 2003, mais il fut mal diffusé à travers le pays. Ce sont les différentes organisations non gouvernementales, 
Cependant, face à la demande, à un manque de personnel formé et à une faible promotion des services mis en place, le dispositif n'a pas porté ses fruits comme l'avait espéré le gouvernement. Ce sont surtout les organismes sociaux locaux, comme IBUKA et AVEGA, qui, grâce à des conseillers formés en traumatisme, ont commencé à prendre en charge les personnes atteintes de troubles psychologiques après une comparution à un gacaca. Ce qui épuise et finit par détruire le rescapé ou le survivant, c'est le fait de devoir assister à répétition à ces juridictions populaires pour entendre les actes barbares des accusés. Pour sa part, Mujawayo a comparu à pas moins de cinq gacaca pour se confronter aux hommes qui avaient massacré sa famille (p. 78). Il est alors indispensable de proposer des structures sociales et psychologiques locales qui aident le rescapé à gérer ses émotions et ses troubles psychiques du moment. Selon Régine King, " the active involvement of the local people and the use of local resources are crucial to the achievement of healing psychological trauma in post-conflict situations and postgenocide Rwanda » (p. 147).

C'est ainsi que, devant les mensonges et les faux-fuyants des divers tueurs de sa sœur comme Étienne, Thomas et Dereva, l'écrivaine, qui, pendant les gacaca, a dû contenir sa colère, soudainement se lâche vers la fin du texte, traitant ces hommes de « salauds de voisins » (2006, p. 224). Il n'y a aucune haine de la part de la narratrice (p. 84, 85 et 127), mais elle a le

comme IBUKA, AVEGA et Médecins sans frontières (MFS) qui vont former des milliers de spécialistes dans le domaine du trauma (Bakansanga et al., p. 4). Esther Mujawayo fait d'ailleurs référence à l'association IBUKA dans La Fleur de Stéphanie (p. 225). Plusieurs autres projets d'encadrement psychosocial des victimes pendant les juridictions gacaca ont vu le jour, dont notamment ceux des institutions kanyarwanda et du Service civil de la paix (ZFD). 
sentiment de revenir « bredouille » (p. 224) après tant d'heures passées à attendre des aveux sincères et une information sur le lieu où se trouve le corps de sa sœur Stéphanie. Qu'est-ce que Mujawayo retire de ces juridictions gacaca: beaucoup d'impuissance (p. 92 et 151), de lassitude (p. 85 et 118) pour elle-même et pour les autres victimes ainsi qu'un retour vers un passé traumatique auquel elles auraient aimé ne pas être confrontées douze ans plus tard. Le ton véhément de Mujawayo est perceptible par l'emploi des points d'exclamation lorsqu'elle défend la cause de toutes les victimes Tutsi vivantes, victimisées lors des juridictions gacaca (p. 225).

\section{Négocier le trauma : le silence, puis la mission}

Après 1994, un silence assourdissant a figé toute la population. Dont les survivants eux-mêmes.

Mujawayo (2006, p. 74)

Après les cris, les pleurs, les plaintes de douleur, les survivants et les rescapés se sont murés dans le silence de leur souffrance. Certains tueurs, eux, se sont cachés derrière un mutisme coupable. De plus, face au risque de retraumatisation ou de représailles, les survivants se sont abstenus de tout commentaire public. Chez Esther Mujawayo, il a fallu quelques années pour pouvoir laisser glisser sa plume sur du papier et révéler au grand jour ce qui la hante, notamment l'image de sa sœur «noyée dans les excréments » (p. 15). D’ailleurs, lors d'un entretien avec Simone Veil, elle ne cache pas la difficulté de passer par la forme écrite pour se raconter : «Le livre que nous venons d'écrire a été très douloureux pour moi parce que j'ai ressenti plus que jamais le manque des miens mais aussi l'intolérable de ce qui nous est arrivé. » (p. 240) De fait, selon 
Arnaud Tellier, « écrire le trauma, ce serait gratter, par feuille de papier interposée, là où ça fait mal. Transposer le trauma, le faire passer de la sphère psychique à la scène scripturale, ne va pas sans réveiller, voire entretenir, le point de douleur psychique. » (p. 89) L'écriture d'une histoire personnelle aussi dramatique permet un meilleur contrôle du trauma puisqu'elle organise et structure la reconstruction de l'être. Mujawayo se voit confrontée à deux éléments : d'une part, les émotions, les images, les souvenirs qui se trouvent refoulés en elle depuis un temps; d'autre part, les mots qui se bousculent mais qui se trouvent organisés par la syntaxe. S'impose aussi la linéarité des faits historiques pour les expliquer aux plus jeunes ou au lecteur peu averti. Le courage du témoin est alors de dévoiler ses émotions par la configuration linéaire de la langue. Chaque détail révélé est pensé, articulé, rectifié pour les besoins de l'édition; ainsi, le sujet traumatisé reprend la maîtrise de ses propres angoisses et de ses souvenirs intrusifs. Pourtant, ce processus symbolique n'est pas sans danger: il arrive que, devant la force du souvenir ou de l'émotion, les mots s'effacent pour faire place au silence. Mais que l'on ne s'y trompe pas, le silence est une forme de discours qui prend différentes formes textuelles. L'auteure s'efforce de s'exprimer, mais devant l'impossibilité de représenter par des mots, quelque chose qui échappe au langage se perd dans l'espace serein du silence. Ainsi, Mujawayo exprime sa réticence à dire par des points de suspension: "elle est, elle est... dans un conduit d'égoût » (p. 15), dit-elle en parlant du lieu où repose sa sœur, ou encore : «c'est visualiser son vi... » (p. 105), n'osant pas finir sa phrase de peur de voir le visage souffrant de Stéphanie. Puis, l'ellipse permet à l'auteure de passer à un autre point sans pour cela conclure son récit : « Remarque, son assassin pourrait peut-être 
m'informer davantage cette fois... Je t'avais raconté qu'un jour j'étais tombée sur lui. » (p. 79) Enfin, pour marquer l'absence totale de mots dans le témoignage de Mujawayo, Belhaddad a donné des repères visuels au lecteur en plaçant à de nombreux endroits dans le texte le mot «Silence » mis entre parenthèses. Parfois, au détour d'un aveu, Mujawayo se perd dans l'émotion, ce qui fait que Belhaddad traduit cela sur le papier par des marques textuelles : " (long silence. Puis, sur un ton confondu, comme pour s'excuser de ne pas retenir son émotion) 》 ou encore, simplement, " (Long silence) », qui est répété pas moins de trois fois dans une seule page alors que Mujawayo revient sur les derniers instants de la vie de Stéphanie (p. 15). Au seuil de l'insupportable sont les images qui se bousculent dans la tête de Mujawayo chaque fois qu'elle s'arrête au sort de sa sœur bien-aimée. À ce stade, les non-dits de l'auteure sont beaucoup plus éloquents que les paroles qu'elles pourraient prononcer. $\mathrm{Au}$ demeurant, elle n'est pas la seule à se réfugier dans le silence, car ces femmes qui font partie des "Réconciliateurs", celles qui ont connu, tout comme elle, la peur de mourir et la souffrance de voir mourir les leurs, réagissent de la même façon par le silence (Mujawayo, 2006, p. 142, 147 et 158).

Une fois que les silences pesants sont passés, la parole ou l'écriture demeure. Mais, parfois, au fond de lui-même, le sujet traumatisé a besoin de bien plus que cela pour se reconstruire. Comme le confirme Judith Herman, certains survivants « discover that they can transform the meaning of their personal tragedy by making it the basis for social action » (p. 207). C'est le cas d'Esther, mais aussi de Théophilia, de Joséphine, d'Odette et de Stéphanie. La mission d'Esther Mujawayo, c'est avant tout son implication dans AVEGA, l'Association des veuves du génocide d'avril, reconnue par les autorités rwandaises depuis 1995, puis dans la 
publication de tous ses livres. Certaines choisissent d'aider d'autres victimes ou encore de se faire inviter à des conférences publiques, comme Esther, tandis que d'autres, préfèrent s'attaquer à la lourde tâche de modifier les comportements des agresseurs, espérant ainsi éviter que l'histoire se répète.

La mission du survivant, qui l'oriente vers une intervention sociale qui dépasse le cadre de ses problèmes personnels, ne doit pas pour autant cacher que la véritable quête du témoin survivant est de retrouver ses morts. Ainsi, parallèlement à leur dévouement à la cause de la reconstruction nationale, ces femmes demeurent, avant tout, des personnes meurtries cherchant dans les tribunaux gacaca le lieu où sont enterrés leurs proches. Certaines, comme Esther et Théophilia, avouent que la seule façon de gérer leur détresse et leur souffrance quotidienne est de devenir une "victime "agissante" » (Mujawayo, 2006, p. 207), de tenir un rôle prépondérant dans la réconciliation demandée par les autorités. Pour Théophilia, être "chargée de suivre les programmes des camps de travaux d'intérêt général, les TIG », lui permet de se confronter aux génocidaires et de se libérer d'eux, mais aussi de "préparer le champ», l'avenir pour les prochaines générations ou encore de "porter un message» (Mujawayo, 2006, p.134-135). Il en va de même pour Joséphine, mère de quatre enfants massacrés, qui se doit de sensibiliser les détenus à la paix pour d'abord se sentir réconciliée avec elle-même, puis se rapprocher des autres (p. 154). Membre d'AVEGA, tout comme Joséphine et Esther, Odette, première vice-présidente en cour d'appel de gacaca, a perdu son mari dans le génocide. Selon elle, «pour préparer l'avenir d'un pays, on ne peut pas toujours rester dans la bagarre; alors, faute de mieux, on accepte le chemin le plus 
court. Mais le plus dur, aussi. » (p. 169) En effet, ce qui ressort des témoignages de ces femmes pleines de courage et de perspectives positives en l'avenir de leur pays, c'est cette résolution qu'il n'y a pas de retour en arrière possible. Fortes, déterminées, elles le sont, mais les images obsédantes de sauvagerie humaine envahissent leur vie quotidienne. Théophilia avoue sincèrement: "Je reste avec la mort en moi, oui, mais j'avance. » (p. 135) Odette, elle, juste et neutre lors des séances gacaca, ne peut empêcher sa colère intérieure d'éclater ou ses larmes de couler une fois chez elle (p. 169 et 174). Ainsi, cette reconstruction par le dialogue avec l'autre dans un cadre contrôlé ne referme pas toutes les blessures. Le fait de s'impliquer dans une cause nationale ou personnelle, comme l'écriture, suggère seulement que le chemin vers la guérison, s'il y en a une un jour, est en cours. En fait, grâce à ces initiatives constructives et libératrices, chaque victime "is ready to take concrete steps to increase her sense of power and control, to protect herself against future danger, and to deepen her alliances with those whom she has learned to trust » (Herman, p. 197).

Nous espérons, dans cette étude, avoir réussi à exposer les effets positifs et négatifs des juridictions gacaca sur les victimes du génocide à travers le témoignage de Mujawayo. Si, d'un côté, ces juridictions ont favorisé la réconciliation en libérant la parole des accusés et des victimes en les autorisant à se souvenir du passé et en essayant de réhabiliter leur histoire personnelle dans un événement collectif national, elles ont aussi réouvert des blessures psychologiques chez les survivants, qui souffraient déjà de l'état de stress post-traumatique (ESPT) ou de dépression. À en juger par le témoignage de Mujawayo, les accusés n’ont pas hésité à rire, à 
ironiser, à humilier ou à mentir devant des victimes prêtes à écouter tous leurs débordements déplacés pour apprendre quelques détails sur la mort des leurs. À cet égard, une étude réalisée par Karen Brounéus montre que "that witnessing may have this worsening effect on depression and PTSD because of the nature of witnessing in truth-telling procedures » (p.429). Selon Brounéus, dire la vérité n'est pas une forme de mécanisme pour la guérison (p.430). C'est plutôt le fait de parler devant un groupe, de se placer en tant qu'individu dans une dynamique de réinsertion sociale qui l'est. Ce sont là les étapes qui prennent une dimension thérapeutique pour le sujet traumatisé. Pour autant, d'autres voies doivent être choisies comme celles des centres d'entraide aux victimes, les thérapies de groupe dans les organismes ou encore les fameuses «missions » que s'imposent les survivants. Les témoins du livre de Mujawayo en sont les parfaits exemples. Ils attendaient beaucoup de ces juridictions et, après l'analyse de leurs témoignages, il est manifeste que cette forme de justice transitionnelle n'a pas refermé les blessures du passé. Bien au contraire, de nombreuses plaies physiques et psychiques restent à être pansées.

Mujawayo a su, avec l'aide de Belhaddad, proposer un texte qui, à travers de multiples stratégies textuelles, met en relief la détermination des victimes de s'en sortir tout en vivant avec cette souffrance intérieure de ne pas avoir pu enterrer, pour beaucoup d'entre elles, leurs morts dignement. Souhaitons que les témoins, comme Esther, continuent d'écrire leur histoire, d'archiver leurs souvenirs et leurs émotions, car la mémoire est, pour reprendre les termes de Georges Kantin, « objet d'étude et objet de transmission volontaire; elle est ce qui demeure quand les acteurs de la tragédie ont disparu » (p. 213). 


\section{Bibliographie}

BAKANSANGA, Appoline, Émilienne MuKAnsoro, Benjamin KumPF, Heide RIEDER et Kathrin GRoninger. (2007), Gacaca et trauma. Le suivi psychosocial des victimes de traumatisme au Rwanda, Ibuka, Kanyarwanda, Service allemand de développement (DED / ZFD), Kigali, <http://www.zivilerfriedensdienst.org/sites/ziviler-

friedensdienst.org/files/anhang/publikation/zfd-gacaca-ettrauma-1502.pdf>.

BARDÈCHE, Marie-Laure. (1999), Le Principe de la répétition, Paris / Montréal, L'Harmattan.

BORNAND, Marie. (2004), Témoignage et fiction. Les récits de rescapés dans la littérature de langue française (1945-2000), Genève, Librairie Droz.

BRounÉUS, Karen. (2010), « The Trauma of Truth-Telling: Effects of Witnessing in the Rwandan Gacaca Courts on Psychological Health », Journal of Conflict Resolution, vol. 54, no 3, p. 408-437.

Collard, Marie-France et Patrick CZAPLinsKi. (2005), Rwanda 94, perf. Yolande MuKAGASANA, enregistré au théâtre de la Place à Liège, Groupov et Parallèles Productions, film, <http://www.youtube.com/watch?v=w006-qa1ffc $>$.

CoQuio, Catherine. (2004), Rwanda. Le réel et les récits, Paris, Belin. HERMAN, Judith. (1992), Trauma and Recovery, New York, Harper Collins.

KANTIN, Georges et Gilles MANCERON. (1991), Les Échos de la mémoire. Tabous et enseignements de la Seconde Guerre mondiale, Paris, Le Monde Éditions. 
KING, Régine. (2011), « Healing Psychosocial Trauma in the Midst of Truth Commissions: The Case of Gacaca in Post-Genocide Rwanda », Genocide Studies and Prevention, vol. 6, n² 2, p. 134-151.

MAINGUENEAU, Dominique. (1994), L'Énonciation en linguistique française, Paris, Hachette.

Mujawayo, Esther et Souâd Belhaddad. (2006), La Fleur de Stéphanie: Rwanda entre réconciliation et déni, Paris, Flammarion.

-. (2004), SurVivantes: Rwanda, dix ans après le génocide, Paris, Éditions de l'Aube.

MUtARABAYIRE SCHAFER, Amélie. (2010), «Du traumatisme du génocide à la violence de la réconciliation. Gestalt-thérapie et soutien psychologique des rescapés du génocide au Rwanda », Cahiers de Gestalt-thérapie, vol. 26, n으. p. 143-162, <http://www.cairn.info/zen.php?ID ARTICLE=CGES 026 0143>.

PoIVRe D'ArVor, Patrick. (2006), entretien télévisé avec Esther Mujawayo, émission littéraire «Vol de nuit », TF1, 28 mars, <http://www.dailymotion.com/video/x4i6xu esthermujawayo-la-fleur-de-stephan news\#.UeaP6ti5p8M>.

Ricceur, Paul. (1986), Du texte à l'action, Paris, Seuil.

SAGARRA, Catalina. (2012), "Texte, contexte et interprétation : visées et modalités du récit de témoignage d'Esther Mujawayo dans SurVivantes », Études littéraires, vol. 43, no 1, p. 107-116.

TELLIER, Arnaud. (1998), Expériences traumatiques et écriture, Paris, Anthropos.

WAintrater, Régine. (2003), Sortir du génocide. Témoignage et survivance, Paris, Petite bibliothèque Payot. 


\section{Résumé}

Dans son récit testimonial La Fleur de Stéphanie (2006), écrit en collaboration avec son amie journaliste Souâd Belhaddad, Esther Mujawayo relate cette période difficile où, en 2005, elle a dû faire face au tueur de sa sœur Stéphanie au Rwanda. Dans cette étude, il s'agit de montrer qu'au travers d'un témoignage dit "élaboratif», Esther Mujawayo fait entendre sa voix traumatisée de victime, mais aussi celles de professionnelles impliquées dans le système de reconstruction nationale. Livrant ses réflexions sur la justice et sur la douleur du souvenir en utilisant divers procédés énonciatifs et discursifs, Mujawayo dévoile les limites et les conséquences (sociales, émotionnelles, psychologiques) des tribunaux gacaca. Peut-on parler de réconciliation quand certains tueurs nient ou mentent? Même si le tueur parle, comment faire face à son mensonge sans tomber dans la folie? Quelle justice paraît adéquate face à l'horreur des révélations? Partagée entre distance et emportement, entre parole et silence, entre guérison et reviviscence du trauma, Esther Mujawayo lutte avec les mots pour se construire un avenir et entretenir « une mémoire contre l'oubli ». 


\begin{abstract}
In her testimonial narrative entitled La Fleur de Stéphanie (2006), written in collaboration with her friend and journalist Souâd Belhaddad, Esther Mujawayo recounts a difficult period where, in 2005, she had to face the killer of Stéphanie, her sister, in Rwanda. In this research paper, I will show that through her "elaborative" testimony, Mujawayo expresses her traumatized voice as a victim as well as those of professionals involved in the national reconstruction of the Rwandan nation. Delivering her reflections on the justice and on the pain of remembering by using diverse enunciative and discursives strategies, Mujawayo reveals the limits and impacts (social, emotional, psychological) of the Gacaca courts. Can we speak about reconciliation when certain killers deny or lie? Even if the killer speaks, how do we confront his lies without falling into madness? What justice seems adequate in front of the horror of the revelations? Torn between distance and fury, word and silence, cure and revival of the trauma, Esther Mujawayo fights with the words to build herself a future and maintain "a memory against forgetting".
\end{abstract}

